\title{
Analog Joint Source Channel Coding Using Space-Filling Curves and MMSE Decoding
}

\author{
Yichuan $\mathrm{Hu}$ and Javier Garcia-Frias \\ ECE Department \\ University of Delaware \\ E-mail: \{yhu, jgarcia\}@ee.udel.edu
}

\author{
Meritxell Lamarca \\ Dept. of Signal Theory and Comm. \\ Technical University of Catalonia (UPC) \\ E-mail: xell@gps.tsc.upc.edu
}

\begin{abstract}
We investigate the performance of a discrete-time all-analog-processing joint sourcechannel coding system for the transmission of i.i.d. Gaussian and Laplacian sources over AWGN channels. In the encoder, two samples of an i.i.d. source are mapped into a channel symbol using a space-filling curve. Different from previous work in the literature, MMSE decoding instead of ML decoding is considered, and we focus on both high and low channel SNR regions. The main contribution of this paper is to show that the proposed system presents a performance very close to the theoretical limits, even at low SNR, as long as the curve parameters are properly optimized.
\end{abstract}

\section{Introduction}

In this paper we consider analog transmission of discrete-time continuous-amplitude sources over additive white Gaussian noise (AWGN) channels. From a theoretical perspective, it is well known that a digital system based on separation between source and channel coding is optimal [1]. Thus, in order to approach the theoretical limit specified by $R_{c} R(D)<C$, where $R_{c}$ is the code rate, $R(D)$ is the rate distortion function, and $C$ is the channel capacity, in traditional communications systems the continuous source is first source encoded (e.g., using very powerful vector quantization) up to the desired rate/distortion pair. Then, capacity approaching channel codes such as turbo codes or LDPC codes are applied.

Provided that both the source encoder and the channel encoder approach optimality, the separated scheme described above will achieve a performance close to the theoretical limits. However, the price to pay is a very high encoding/decoding complexity and significant delays, since any capacity approaching channel code requires long block lengths. Furthermore, such separated system has to be specifically designed for the desired rate and distortion: if the desired rate/distortion pair changes, the system has to be completely re-designed.

Interestingly, it is well known that under some circumstances analog communications are optimal. For instance, direct transmission of uncoded Gaussian samples over AWGN channels is optimal [2]. In that sense, it is said that Gaussian sources are perfectly matched to Gaussian channels [3]. Therefore, it is not surprising that previous work in the literature has investigated possible schemes based on analog transformations aiming at perfectly "matching" sources with channels. However, although some promising schemes have been proposed $[4,5,6,7]$, among others, research in this area is still in its infancy. 
Among the few practical analog coding schemes that have appeared in the literature, those based on the use of space-filling curves, already proposed more than 50 years ago by Shannon [8] and Kotel'nikov [9], have recently acquired a renewed importance due to the work of Fuldseth [4], Chung [5], Ramstad [6] and Hekland [7]. The encoding idea to reduce the number of samples to be transmitted is to represent a tuple of $n$ source samples as a point in an $n$-dimensional space where a spacefilling surface of dimension $k$ lives. Then, the $n$-tuple is projected onto the curve and the corresponding $k$-tuple is transmitted through the noisy channel. Maximum Likelihood (ML) or Minimum Mean Squared Error (MMSE) decoding is performed to recover the original data.

Most of the work on space-filling curves has focused on ML decoding and high signal to noise ratios (SNR). In these conditions, it is possible to analyze the system performance, and use this analysis to optimize the curve parameters [5, 7]. For diverse sources and high SNR, ML decoding results in a performance very close to the theoretical limits. This is extremely interesting, since the system complexity is much lower than that of a separated scheme. Moreover, since no long blocks are used the delay is very small. However, ML decoding does not perform well for low SNR, and even in the case of high SNR it is not optimal. The case of MMSE decoding has been mentioned in [5] for uniform sources and Hilbert-like curves. However, in order to analyze/optimize performance in high SNR situations, [5] focuses on suboptimal MMSE decoding, which results in performance degradation for low SNR. To our knowledge, quasi-optimal MMSE decoding and optimization for all SNR values has not appeared in the literature. The common understanding about these mappings is that they perform quite well for high SNR, but experience significant degradation for low SNR.

In this paper, we present a technique to perform quasi-optimal MMSE decoding of i.i.d. Gaussian and Laplacian sources transmitted over AWGN channels, and optimize the curve parameters to obtain a performance that is very close to the theoretical limits in the whole SNR region. We focus on 2:1 mappings, which are the most commonly studied in the literature, but these techniques can easily be extended to other mappings. As we will see in the sequel, the performance improvement with respect to ML is very significant, and, contrary to ML decoding, the gap with respect to the theoretical limit gets smaller when SNR decreases.

\section{System Model}

\subsection{Encoder}

The key problem in the encoder is the design of a space-filling curve that is appropriate for the source distribution and the channel SNR of interest. As explained before, the use of space-filling curves was proposed in $[8,9]$. Their importance in the context considered here was corroborated in [4], where power constrained channel optimized vector quantization (PCCOVQ) was studied. The results obtained in [4] when the PCCOVQ codebook is optimized are excellent for low SNR (about $1 \mathrm{~dB}$ away 
from the theoretical limit for Gaussian sources transmitted over AWGN channels). However, as the channel SNR increases, it is necessary to train very large codebooks, which makes the PCCOVQ optimization process very complex and leads to significant performance degradation. Obviously, by connecting the adjacent vectors in the PCCOVQ codebook we can obtain a space-filling curve, which, interestingly, for moderate to high SNR, coincides with the ones previously proposed in $[8,9]$. In that sense, [4] justifies the use of space-filling curves for the problem of joint source-channel coding.

The direct use of parameterized space-filling continuous curves rather than PCCOVQ approaches eliminates the problem of codebook optimization, which is intractable for very high SNR. The renewed interest on these schemes began with the work of Chung [5] and Ramstad [6]. Spiral-like and deformed spiral-like curves were respectively proposed for transmission of Gaussian and Laplacian sources over AWGN channels, while Hilbert (and similar) curves were applied for transmission of uniform sources over modulo AWGN channels.

In this paper, we focus on spiral-like curves, which can be defined in the following parametric form [7]

$$
\left\{\begin{array}{l}
x=\frac{\Delta}{\pi} \theta \sin \theta \\
y=\frac{\Delta}{\pi} \theta \cos \theta
\end{array} \text { for } \theta \geq 0, \quad\left\{\begin{array}{l}
x=-\frac{\Delta}{\pi} \theta \sin \theta \\
y=\frac{\Delta}{\pi} \theta \cos \theta
\end{array} \text { for } \theta<0,\right.\right.
$$

where $\Delta$ is the distance between two neighboring spiral arms and $\theta$ is the angle from the origin to the point $(x, y)$ on the curve. We will see that the appropriate use of these curves results in excellent performance for both Gaussian and Laplacian sources.

We will also consider the deformed spiral-like curve proposed in [10] for Laplacian sources

$$
\begin{aligned}
& \left\{\begin{array}{l}
x=\frac{\Delta}{\pi} \theta^{1 / k}\left[\sin \left(\theta-\frac{3 \pi}{16}\right)+m \cos (3 \theta)\right] \\
y=\frac{\Delta}{\pi} \theta^{1 / k}\left[\cos \left(\theta-\frac{3 \pi}{16}\right)+m \sin (3 \theta)\right]
\end{array} \text { for } \theta \geq 0,\right. \\
& \left\{\begin{array}{l}
x=\frac{\Delta}{\pi} \theta^{1 / k}\left[\sin \left(-\theta-\frac{3 \pi}{16}\right)+m \cos (-3 \theta)\right] \\
y=\frac{\Delta}{\pi} \theta^{1 / k}\left[\cos \left(-\theta-\frac{3 \pi}{16}\right)+m \sin (-3 \theta)\right]
\end{array} \text { for } \theta<0 .\right.
\end{aligned}
$$

Instead of one parameter as in (1), the number of parameters in this case is three. This increase in degrees of freedom allows a better match to the optimal PCCOVQ codebook for Laplacian sources. However, it also makes the optimization more complex. In fact, one of the interesting results in this paper is that under MMSE decoding, and as long as the parameters are properly chosen, the use of simpler spirallike curves for the encoding of Laplacian sources performs as well as the deformed one. 


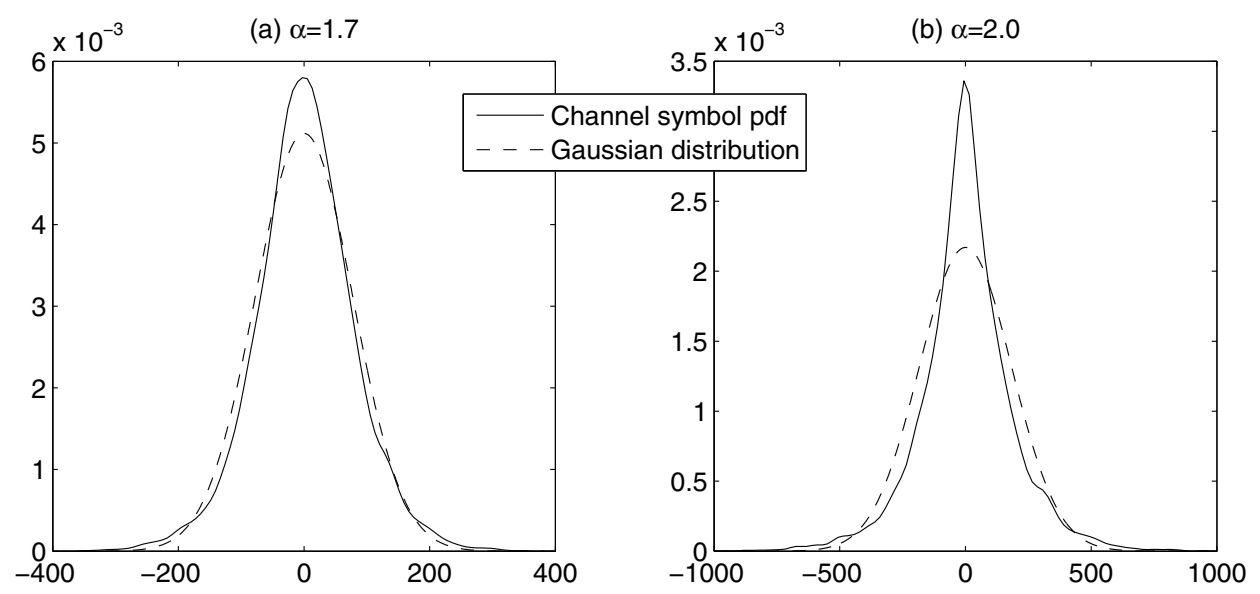

Figure 1: For the spiral-like curve and Gaussian sources, probability density function (pdf) of the input channel symbols before normalization, $T_{\alpha}(\hat{\theta})$, when $\Delta$ is optimized for $\alpha=2$ using the high SNR approximation from [5, 7] for channel SNR $=10 \log \frac{1}{\sigma_{n}^{2}}=40 \mathrm{~dB}$, and (a) $\alpha=1.7$, (b) $\alpha=2$. The Gaussian approximation of these data is also shown.

Notice that in the two curves described above there is a one-to-one correspondence between parameter $\theta$ and the points $(x, y)$ on the curve, so that the curve gradually fills in the whole $x$ - $y$ plane as the absolute value of $\theta$ grows. Therefore, we can use a mapping function to project a pair of source samples $(x, y)$ onto the curve by finding the closest point on the curve. For the spiral-like curve, the mapping function $M_{\Delta}(\cdot)$ is defined as

$$
\hat{\theta}=M_{\Delta}(x, y)=\arg \min _{\theta}\left\{\left(x \pm \frac{\Delta}{\pi} \theta \sin \theta\right)^{2}+\left(y-\frac{\Delta}{\pi} \theta \cos \theta\right)^{2}\right\} .
$$

Then, an invertible function of $\hat{\theta}$, normalized so that the average transmitted energy per sample is equal to 1 , is transmitted through the AWGN channel. In order to do so, we introduce a simple transform function $T_{\alpha}(x)=x^{\alpha}$ where $\alpha \in(0,2]$ is a parameter that has to be optimized for different channel SNR, and denote the symbols transmitted through the channel as $T_{\alpha}(\hat{\theta}) / \sqrt{\gamma}$, where $\sqrt{\gamma}$ is a normalization factor so that the average energy per sample transmitted through the channel is equal to 1 . In all previous work in the literature $[5,7], T_{2}(x)=x^{2}$ is considered, since in this manner the transmitted value $\hat{\theta}^{2}$ is proportional to the length of the curve and its probability density function (pdf) at high SNR is Laplacian, which facilitates performance analysis (the only exception is [11], where the use of $\alpha=1$ was shown to lead to some performance degradation). However, we will see in the sequel that transmitting $\hat{\theta}^{2}$ does not guarantee optimality. In fact, in order to achieve the capacity of AWGN channels, transmitted symbols should follow a Gaussian distribution, and choices of $\alpha$ different from 2 can lead to a better Gaussian approximation. This is illustrated in Figure 1, which shows the pdf of the symbols transmitted through the channel for different values of parameter $\alpha$.

In sum, the received observation at the decoder can be expressed (see Figure 2) as $r=T_{\alpha}\left(M_{\Delta}(F(x, y))\right)+\sqrt{\gamma} n$, where $F(x, y)$ is a transformation of the i.i.d. samples $x$ and $y$, and $n \sim \mathcal{N}\left(0, \sigma_{n}^{2}\right)$ is additive white Gaussian noise. Transformation $F(\cdot)$ is used 


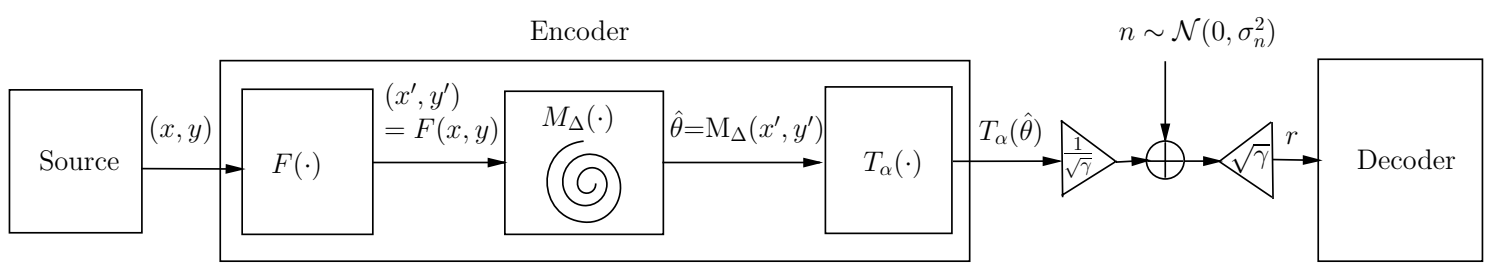

Figure 2: System model.

in the case of Laplacian sources when spiral-like curves are applied, and transforms a Laplacian random variable into a Gaussian random variable. The rationale is that spiral-like curves are originally designed to achieve good performance in the case of Gaussian sources. However, we will see in the sequel that when MMSE is used and curve parameters are optimized, spiral-like curves also achieve good performance in the case of Laplacian sources, and thus transformation $F(\cdot)$ is unnecessary.

\subsection{Decoder}

\subsubsection{Decoding}

Given a received symbol $r$, the ML estimate is obtained as the source pair $(x, y)$ belonging to the curve and satisfying

$$
\left\{\hat{x}_{M L}, \hat{y}_{M L}\right\}=\underset{(x, y) \in \text { curve }}{\arg \max }\{p(r \mid x, y)\}=\left\{x, y \mid(x, y) \in \text { curve and } T_{\alpha}\left(M_{\Delta}(F(x, y))\right)=r\right\} .
$$

ML decoding is equivalent to first applying the inverse function $T_{\alpha}^{-1}(\cdot)$ to received symbol $r, \hat{\theta}^{\prime}=T_{\alpha}^{-1}(r)=r^{-\alpha}$, and then performing inverse mapping on $\hat{\theta}^{\prime}$ according to $(1)$ or $(2)$.

\subsubsection{MMSE Decoding}

Although ML decoding is simple and performs very well at high SNR, it is not optimal for the mean square error distortion criterion (MSE). In this case, MMSE decoding is optimal, and can be expressed as

$$
\begin{aligned}
\left\{\hat{x}_{M M S E}, \hat{y}_{M M S E}\right\} & =E\{x, y \mid r\}=\iint\{x, y\} p(x, y \mid r) \mathrm{d} x \mathrm{~d} y \\
& =\frac{1}{p(r)} \iint\{x, y\} p(r \mid x, y) p(x) p(y) \mathrm{d} x \mathrm{~d} y .
\end{aligned}
$$

Since the conditional probability $p(r \mid x, y)$ involves the mapping function $M_{\Delta}(\cdot)$ which is discontinuous and highly non-linear, (5) can only be calculated numerically. We utilize a very simple but accurate way to approximate the above integral. First, we discretize the $x-y$ plane using a uniform step, and calculate a mapped value for each discretized point according to (3). By doing so, we obtain a discretized version of $p(r \mid x, y)$. Then, by applying the same discretization procedure to $x, y, p(x)$ and $p(y)$, the calculation of the above integral is simplified to only multiplication and addition 


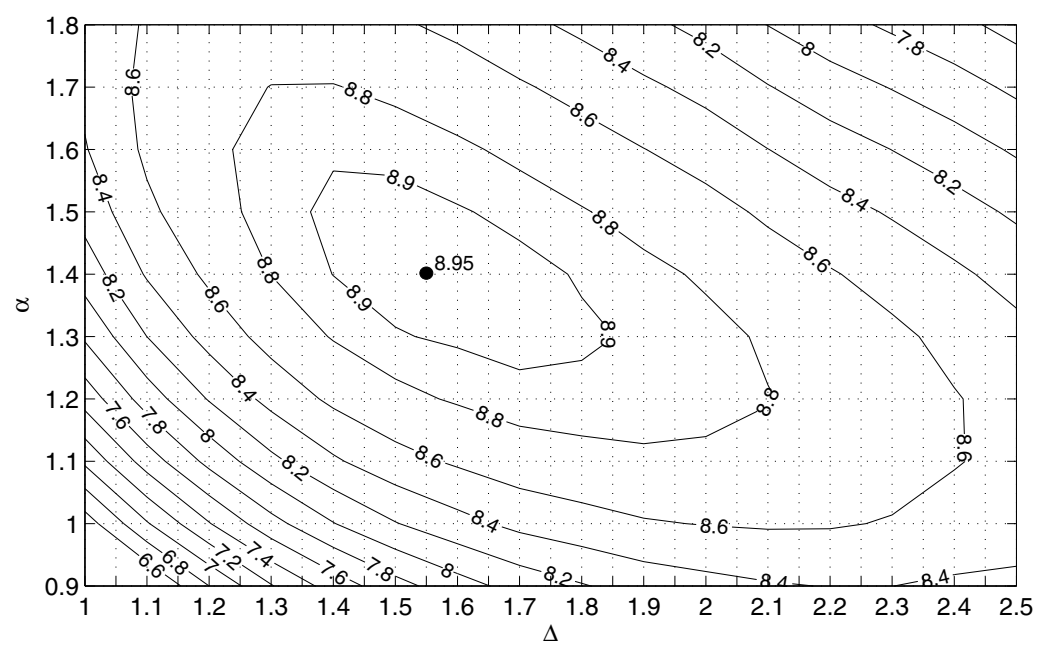

Figure 3: Level curves showing the output SDR as a function of parameters $\Delta$ and $\alpha$ in the spiral-like curve for a Gaussian source when ML decoding is used and the channel SNR = $20 \mathrm{~dB}$.

operations. Moreover, since the discretization step does not depend on the received symbol, it only needs to be done once off-line and stored in the decoder. Thus, the complexity of MMSE decoding is greatly reduced.

\section{Parameter Optimization}

As described in previous section, the encoder is fully characterized by the curve parameters ( $\Delta$ for the spiral curve) and $\alpha$. Thus, given the source distribution, the channel SNR, and a decoding technique (ML or MMSE), the system performance only depends on the curve parameters, which should therefore be optimized.

\subsection{Optimization for ML}

As described in [5, 7], for ML decoding and high SNR the overall distortion when $\alpha=2$ is minimized by balancing the so called approximation and channel distortions, so that the optimal parameter $\Delta$ in spiral-like curves can be obtained as a closed function of $\mathrm{SNR}^{1}$. However, if $\alpha \neq 2$ or $\mathrm{SNR}$ is low, the value of $\Delta$ obtained in this manner is no longer optimal. This is corroborated in Figure 1, which shows that simply changing $\alpha$ from 2 to 1.7 makes the symbol distribution closer to Gaussian. Simulations corroborate that this brings in a $0.2 \mathrm{~dB}$ improvement in the output signal-to-distortion ratio $\left(S D R=10 \log \frac{\sigma_{x}^{2}}{M S E}\right)$ for a channel SNR of $40 \mathrm{~dB}$.

As described before, optimization needs to be performed for all parameters $(\Delta$

\footnotetext{
${ }^{1}$ For both ML and MMSE decoding, the approximation distortion is the reconstruction error that appears because the curve does not fill the space completely, and it is present even if the channel is noiseless. The channel distortion is due to the channel noise, and it is present even if the source samples lie on the space-filling curve. A higher $\Delta$ leads to higher approximation distortion but to lower channel distortion.
} 
(a) ML decoding: $\alpha=1.5, \Delta=3.0, \mathrm{MSE}=0.46$

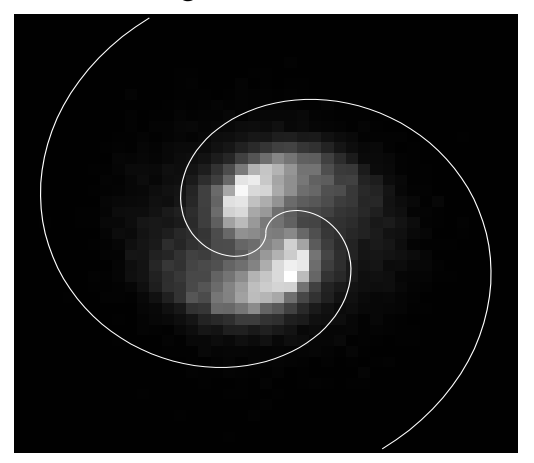

(b) MMSE decoding: $\alpha=1.1, \Delta=3.8$, MSE $=0.36$

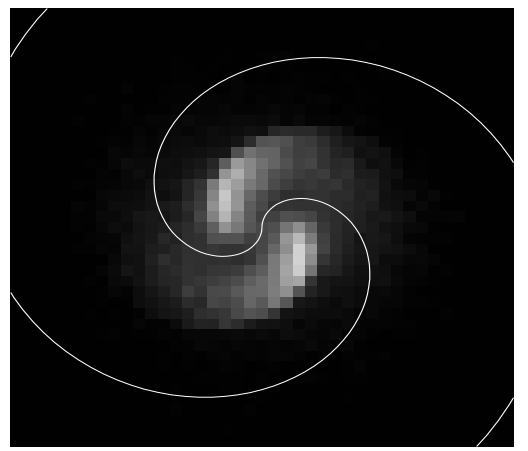

Figure 4: For each point in the $x-y$ plane, average distortion (MSE) when spiral-like curves are used to encode Gaussian sources and the channel SNR is $10 \mathrm{~dB}$. The optimal parameters for a) ML decoding and b) MMSE decoding are considered. The distortion is visualized by grayscale levels, with whiter points indicating larger distortion.

and $\alpha$ in spiral-like curves) and for each one of the values of SNR. Since the input channel symbol does not have a closed distribution when $\alpha \neq 2$, we cannot perform the optimization analytically. Instead, the optimization is done numerically by calculating through simulation the output $\operatorname{SDR}$ for different $(\Delta, \alpha)$ pairs. Figure 3 shows this process for the case of a Gaussian source when the channel SNR is $20 \mathrm{~dB}$. Notice that the optimal parameters $(\Delta=1.55, \alpha=1.4)$ are far away from the ones theoretically obtained using the high SNR analysis in $[5,7](\Delta=1.24, \alpha=2)$. This is intuitive: if we fix $\Delta$ and change $\alpha$ from 0 to 2 , since the distribution of the input channel symbol is a delta function when $\alpha$ equals to 0 and a Laplace distribution for $\alpha$ equal to 2 , there must exist certain $\alpha$ between 0 and 2 that makes the input channel symbol distribution as close to Gaussian as possible. From the level curves of Figure 3, it is interesting to remark that if there is a mismatch in parameters $\Delta$ and $\alpha$, it is better to choose them greater (rather than smaller) than the optimal ones.

\subsection{Optimization for MMSE}

In ML decoding, decoded points are restricted to lie on the space-filling curve. In MMSE decoding, however, decoded points can be at any place in the source plane, which allows to reduce the approximation distortion. Specifically, for a received observation, the MMSE solution is always closer to the origin when compared to the ML decoded point. The reason is that, as indicated in (5), MMSE decoding is the expected value of a density in which a priori information of the source is involved, and Gaussian and Laplacian sources are concentrated around 0. Again, it is not possible to perform the optimization analytically, and we must perform it numerically as described in previous section.

Intuitively, it is easy to see that the parameters optimized for ML decoding are no longer optimal for MMSE decoding. Since MMSE decoding is able to reduce the approximation distortion in spiral-like curves, it can obtain the same approximation distortion as ML with a higher value of $\Delta$. Notice, however, that a higher $\Delta$ leads to lower channel distortion (see footnote 1 ), so that the overall distortion is reduced. 


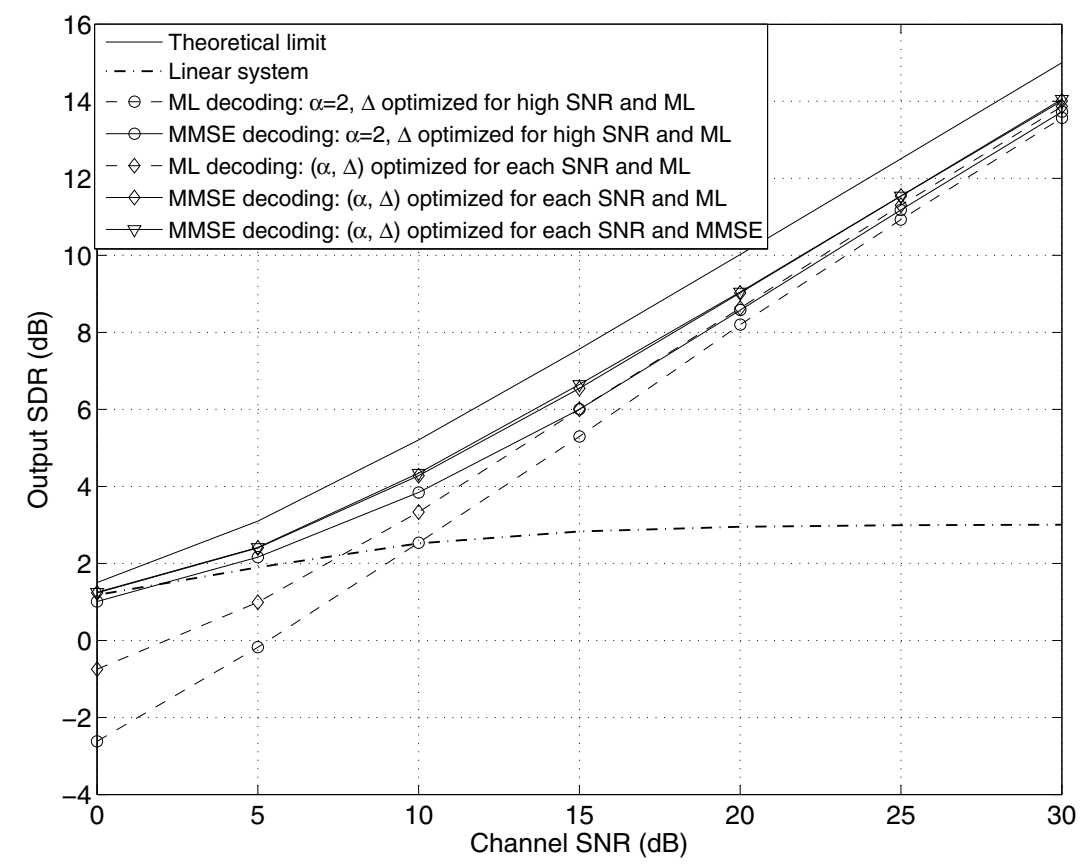

Figure 5: Performance of the proposed system for the transmission of Gaussian sources over AWGN channels.

This is illustrated in Figure 4 for Gaussian sources and spiral-like curves when the channel SNR is $10 \mathrm{~dB}$ : even for a larger value of $\Delta$ (3.8 versus 3.0 in ML decoding), MMSE decoding results in less overall distortion $(\mathrm{MSE}=.36$ versus $\mathrm{MSE}=.46$ in $\mathrm{ML}$ decoding). This is reflected graphically in the existence of less white points around the origin (the region with the most likely source pairs) for the case of MMSE decoding, shown in Figure 4 (b).

\section{Simulation Results}

In our first set of simulations, we consider the transmission of Gaussian sources over AWGN channels using the spiral-like curves defined in (1) and compare it with the linear system in [12]. Without loss of generality, sources with zero mean and unit variance are considered. Figure 5 shows the output SDR versus the channel SNR when MMSE and ML decoding are applied and the curve parameters, $\alpha$ and $\Delta$, are optimized using several criteria. The worst performance is obtained when ML decoding is applied and the curve parameters are chosen applying the high SNR analysis developed in $[5,7]$. In this case, the system behavior is quite good for high SNR, but the performance degrades significantly for low SNR, so that the linear system performs better for SNR below $10 \mathrm{~dB}$. When parameters are optimized following the technique presented in previous section (still with ML decoding), we can observe a performance gain in output SDR of around $1-2 \mathrm{~dB}$ for low $\mathrm{SNR}$ (SNR $\in[0,10]$ $\mathrm{dB}$ ). Additional gains in this low SNR region of around $0.4-2 \mathrm{~dB}$ are obtained if MMSE is used instead of ML decoding. In this case, Figure 5 shows that parameter optimization is less critical than in ML decoding, and the best performance is ob- 


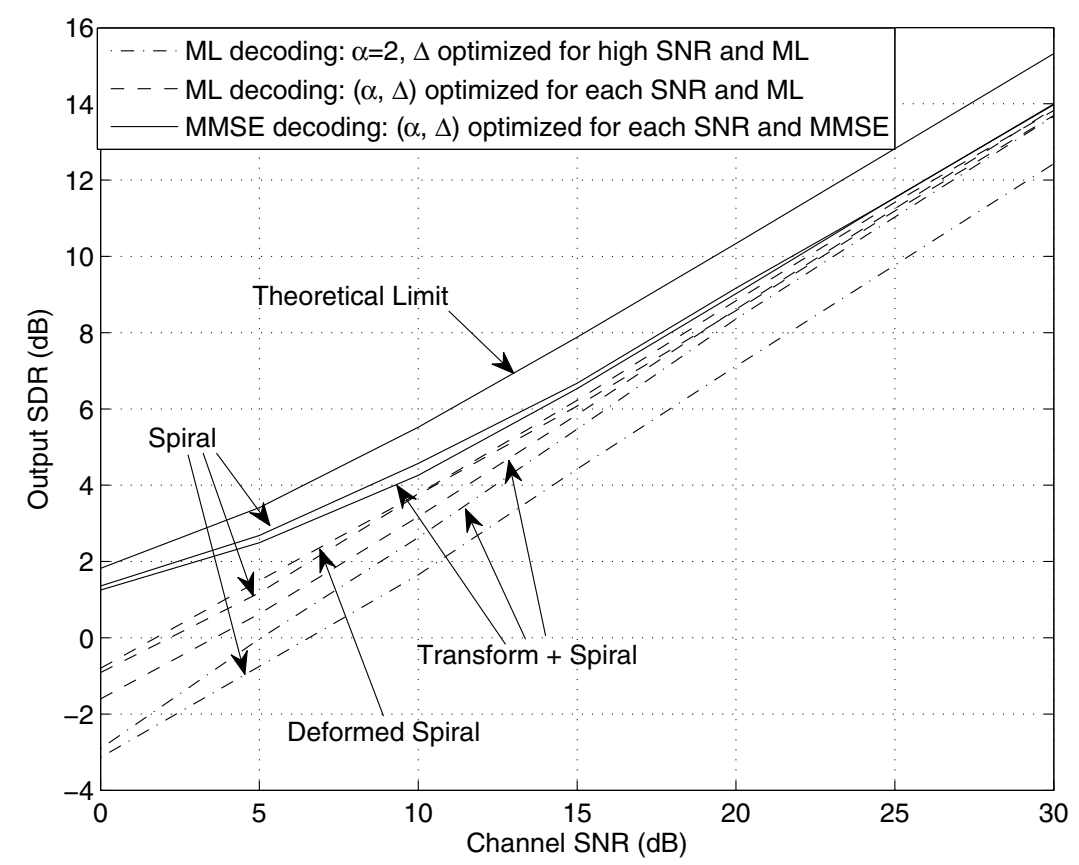

Figure 6: Performance of the proposed system for the transmission of Laplacian sources over AWGN channels.

tained by applying MMSE decoding and optimizing the parameters for this case. It is remarkable that in this situation the gap with respect to the theoretical limit is less than $1 \mathrm{~dB}$ for all values of SNR. However, contrary to the case of ML decoding, when MMSE decoding is applied the gap gets smaller as the channel SNR decreases, and for very low SNR performance is similar to that of a linear system. This occurs because parameter optimization for low SNR makes the space-filling curve approximate a linear system, which is optimal when channel SNR goes to $-\infty$ [12].

We next consider the transmission of Laplacian sources over AWGN channels. Figure 6 presents the resulting performance when either ML or MMSE decoding is applied and three types of encoders are considered: i) spiral-like curves as defined in (1), ii) deformed spiral-like curves (2), which in [10] were shown to present good performance for high SNR and ML decoding, and iii) transformation block (from Laplacian to Gaussian) followed by a spiral-like curve, also proposed in [10]. As explained in section 2.1, the reason for applying this transformation is to take advantage of the good performance that spiral-like curves produce for Gaussian sources. In each case, the system parameters were optimized for the corresponding conditions. It is remarkable that all encoders work very well when MMSE decoding is applied (the optimization process for system ii) is extremely complex in the case of MMSE decoding, and thus it has not been considered here). In fact, although spiral-like curves were originally proposed for Gaussian sources, they are the best system when MMSE decoding is applied: about $1.4 \mathrm{~dB}$ gap to the theoretical limit for high SNR, and smaller gaps for low SNR. Interestingly, when ML decoding is used and the parameters are optimized using the high SNR analysis from [5, 7], the performance of the system based on spiral-like curves is much worse when the transformation block is not used. 


\section{Conclusion}

We have presented a discrete-time all-analog-processing joint source-channel coding scheme, based on the use of spiral-like curves, achieving 2:1 bandwidth reduction for the transmission of i.i.d. Gaussian and Laplacian sources over AWGN channels. By utilizing MMSE decoding and numerically optimizing the curve parameters for each SNR, the proposed system outperforms previous existing schemes in the literature. For the whole SNR range, the gap of the proposed system with respect to the theoretical limit is less than $1 \mathrm{~dB}$ in the case of Gaussian sources, and less than 1.4 $\mathrm{dB}$ for Laplacian sources. Although we have presented these techniques for the case of 2:1 bandwidth reduction, they can easily be applied for other reduction factors.

\section{Acknowledgments}

This work was supported in part by NSF Award ECCS-0725422.

\section{References}

[1] C. E. Shannon, "A mathematical theory of communication," The Bell System Technical Journal, vol. 27, pp. 379-423, 1948.

[2] T. J. Goblick, "Theoretical limitations on the transmission of data from analog sources," IEEE Trans. on Information Theory, vol. 11, no. 4, pp. 558-567, October 1965.

[3] M. Gastpar, B. Rimoldi, and M. Vetterli, "To code, or not to code: lossy source-channel communication revisited," IEEE Trans. on Information Theory, vol. 49, no. 5, pp. 1147-1158, May 2003.

[4] A. Fuldseth and T. A. Ramstad, "Bandwidth compression for continuous-amplitude channels based on vector approximation to a continuous subset of the source signal space," Proc. ICASSP'97, April 1997.

[5] S. -Y. Chung, "On the construction of some capacity-approaching coding schemes," Ph.D. dissertation, Dept. EECS, Massachusetts Institute of Technology, 2000.

[6] T. A. Ramstad, "Shannon mappings for robust communication," Telektronikk, vol. 98, no. 1, pp. 114-128, 2002.

[7] F. Hekland, G. E. Oien, and T. A. Ramstad, "Using 2:1 Shannon mapping for joint sourcechannel coding," Proc. DCC'05, March 2005.

[8] C. E. Shannon, "Communication in the presence of noise," Proc. IRE, vol. 37, pp. 10-21, January 1949.

[9] V. A. Kotel'nikov, "The theory of optimum noise immunity," New York: McGraw-Hill, 1959.

[10] F. Hekland, "On the design and analysis of Shannon-Kotel'nikov mappings for joint sourcechannel coding," Ph.D. dissertation, Norwegian University of Science and Technology, 2007.

[11] A. Ingber and M. Feder, "Power Preserving 2:1 Bandwidth Reduction Mappings," Proc. $D C C^{\prime} 07$, March 2007.

[12] K.-H. Lee and D. P. Petersen, "Optimal linear coding for vector channels," IEEE Trans. on Communications, vol. 24, no. 12, pp. 1283-1290, December 1976. 